Open Access

\title{
On the notion of abstraction in systemic functional linguistics
}

\author{
Jamie Williams ${ }^{1,2^{*}}$ (D), Noah Russell ${ }^{2}$ and Derek $\mid$ rwin $^{3}$
}

\author{
* Correspondence: \\ Jamiewilliams0903@gmail.com \\ 'School of English, University of \\ Nottingham Malaysia Campus, Jalan \\ Broga, 43500 Semenyih Selangor \\ Dahrul Ehsan, Malaysia \\ ${ }^{2}$ School of Electrical and Electronic \\ Engineering, University of \\ Nottingham, University Park, \\ Nottingham NG7 2RD, United \\ Kingdom \\ Full list of author information is \\ available at the end of the article
}

\begin{abstract}
This article provides an in-depth analysis of the theoretical dimensions of stratification, instantiation, and delicacy within Systemic Functional Linguistics (SFL). It does so by examining the nature of the concept of 'abstraction' with respect to these terms. The claim is that, although the same term is used in each case, the precise meaning of abstraction differs depending on the theoretical focus. This conclusion is reached by examining three related, but distinct, factors: (1) omission of detail, (2) generalisation, and (3) decontextualisation. By using these criteria, this article develops a typology of abstraction types for SFL. The conclusion reached is that, although the dimensions of delicacy and instantiation can be described using the criteria above, the explicit ordering behind the dimension of stratification cannot. The paper concludes by examining a proposal that attempts to account for the explicit ordering between strata in terms of supervenience. We argue that this attempt fails to accurate describe inter-stratal relations in the theory. Therefore, the basis of this ordering is in need of further investigation.

Keywords: Abstraction, Systemic functional architecture, Stratification, Instantiation, Delicacy
\end{abstract}

\section{Introduction}

Systemic Functional Linguistic theory employs a theoretical architecture more elaborate than other linguistic theories. Whereas the trend in Generative Linguistics has been to aim for increased parsimony in theoretical constructs since the advent of the Minimalist Program (Chomsky 1995), SFL practitioners argue that a complex theoretical architecture is needed to reflect the equally complex nature of language itself (Matthiessen 2007b; Halliday 2009). In doing so, SFL highlights five fundamental dimensions for studying language: rank, delicacy, stratification, instantiation, and metafunction (Halliday and Matthiessen 2014: 20). Martin (2009) also proposes the dimension of individuation.

This article focuses on three of these: delicacy, stratification, and instantiation, since they have all been associated with the theoretical notion of abstraction. For stratification, Halliday and Matthiessen (1999: 4) state that the strata of phonology, lexicogrammar, and semantics are "differentiated according to order of abstraction". Indeed, stratification is often linked to the notion of abstraction in SFL (see, for example, Halliday 1961, 1981[2005], Hasan 1995, 2013, Matthiessen 2007a., Taverniers 2011, and Martin 2014). Instantiation has also been linked to the notion of abstraction: both in

(c) The Author(s). 2017 Open Access This article is distributed under the terms of the Creative Commons Attribution 4.0 International License (http://creativecommons.org/licenses/by/4.0/), which permits unrestricted use, distribution, and reproduction in any medium, provided you give appropriate credit to the original author(s) and the source, provide a link to the Creative Commons license, and indicate if changes were made. 
Halliday's (1961) foundational work, but more explicitly, Matthiessen (2012: 450) makes the connection in a comparison of SFL with Fairclough's Critical Discourse Analysis. Similarly, the dimension of delicacy is described by Halliday (1961) as operating over a scale of abstraction. Furthermore, Halliday and Matthiessen (1999: 327) state that these three dimensions each refer to a "distinct scale of abstraction". It is clear, then, that abstraction is important when considering the theoretical architecture of SFL.

The motivation for this paper is that abstraction is known to take multiple meanings. Barsalou $(2003,2005)$ gives six possible facets of the term as related to human cognitive capacities, while Saitta and Zucker (2013: Ch.2) identify five possible features as evident from the term's use in a variety of different disciplines, from philosophy and art, to mathematics and computer science. The meanings they identify are (ibid: 47):

- Abstraction is to take a distance from the concrete world.

- Abstraction coincides with (or is a close variant of) generalisation.

- Abstraction is information hiding.

- Abstraction is to keep relevant aspects and to disregard irrelevant ones.

- Abstraction is a kind of reformulation or approximation

Due to this multi-valance, it is important to make certain what is meant by the term's use on a case-by-case basis. Otherwise, misunderstandings concerning the nature of this theoretical notion may arise, and any inexplicitness may hinder the successful application of theoretical notions to real-life cases.

This article argues that although the term abstraction has been applied to all three of the theoretical dimensions mentioned, the specific nature of the term differs in each case. Proceeding from a discussion of abstraction from other academic disciplines, this investigation will focus on the following aspects of abstraction that seem most relevant for functional linguistics: (1) abstraction as linked to the idea of 'mere omission' (c.f. Jones 2005: 174), (2) abstraction as generalisation, and (3) abstraction as decontextualisation. Using these factors as criteria, it is possible to show how stratification, delicacy, and instantiation differ from and are alike to each other in these regards. In doing so, it is hoped that a deeper and clearer understanding of these crucial theoretical notions will be achieved.

The rest of the article is organised as follows: Section 2 provides a discussion of the methodology taken in this work, namely that making a comparison with the use of abstraction in other disciplines can aid our understanding of the term in SFL. It will also address some potential criticisms that may be levied against this approach. Section 3 gives an overview of the facets of abstraction mentioned above. Section 4 discusses the relevant theoretical dimensions in SFL, and investigates the meaning of abstraction in each case. It concludes with the main finding of this study, by giving a typology of the different kinds of abstraction found in systemic theory. Section 5 returns to the notion of stratification, because, as will be shown, this dimension does not map on well to the factors previously discussed. Section 6 concludes the work and points towards further potential avenues of research.

\section{Systemic functional linguistics and the (other) sciences}

Abstraction is considered one of the fundamental concepts in scientific practice (Godfrey-Smith, 2009: 46). Physicists' equations, chemical symbols, and mathematical 
descriptions all involve some degree of abstraction in their creation. As such, the nature of this process has attracted discussion from those working in the philosophy of science, whose aims include investigating the reasoning implicit in the creation of scientific models.

Crucial to the work presented here is a comparison of how abstraction is defined in other disciplines, mainly from the mathematical and physical sciences, with how the term has been used by SFL practitioners. Before commencing with this enterprise, there are potential criticisms with this approach that need to be addressed. Halliday (1992a. [2005]) argues that the objects of scientific study and linguistic study may be of a different type; although there may be some similarities in systems of all kinds, there will undoubtedly be features particular to each discipline. If the subject matter is indeed different, then the question remains whether any comparisons of theoretical terms can prove useful. Secondly, he argues that philosophy of science produces a highly idealised viewpoint, far removed from the daily practices of scientists as they carry out their work. As such, linguists can learn from scientists, if linguists so wish, by observing scientists actually going about their work, and not "studying the models constructed in the name of philosophy of science" (ibid: 200). Drawing upon the work of such writers, then, may be less useful than it may at first seem.

This paper takes the view, however, that although it cannot be assumed that theoretical notions do transcend discipline boundaries, neither can the possibility be dismissed out of hand. As mentioned, abstraction has already been compared in various subjects, and certain regularities have been argued for. If: (a.) the processes that are involved are linked to general properties of human cognition, as has been argued for by Barsalou (2003, 2005) and Martinez and Huang (2011), and (b.) these same capacities are utilised to some degree in all human academic endeavour, as seems reasonable to assume, then there is good reason for thinking that some comparison may prove beneficial, regardless of any purported differences in the systems under study.

Additionally, far from models being constructed "in the name of philosophy of science", idealised models are more often than not constructed by scientists themselves on the basis of experiments performed and observations made. For instance, the abstract, idealised objects which dominate physics are not created by or for the sake of philosophers; they are created so that scientific practitioners can allow their observations to lead to predictions that hold in a wider set of cases. The aim of philosophy of science, or at least the part which interests us here, is therefore to study the kinds of processes involved in the construction of such models. As one example, Cartwright (1983) discusses the difficulties in linking mathematical laws in physics with the real world, by highlighting the problems of marrying the certus paribus foundations of physical description with the multitude of causes which may act to produce a certain effect in reality. ${ }^{1}$ Her argument is, briefly, that generalised laws in physics do not state facts about how 'real' objects behave, but instead how theoretical objects behave within models, which are usually highly idealised. Regardless of whether Cartwright's views are correct, and there have been many replies to her arguments, the important issue for this piece is that scientific models, which would include theoretical constructs such as laws, far from being constructed "in the name of philosophy of science" are more often than not constructed by scientists in the name of science. Indeed, this is much a part of their profession as the rigorous, methodological procedure that is the hallmark of 
scientific experimentation. The role for those interested in investigating scientific practice is then to enquire into the processes involved for its creation, instead of creating models for their own sakes. This would hold for theoretical constructs in a more socially-orientated theory such as SFL, as it would for the more mathematically orientated disciplines.

With these potential criticisms addressed, it is now possible to outline the different meanings that have been associated with abstraction in other disciplines.

\section{The different aspects of abstraction}

\section{Abstraction as 'mere omission'}

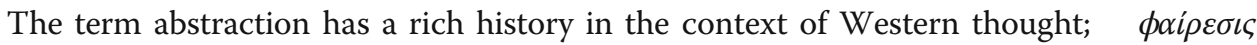
(abstraction) is discussed in both Aristotle's Analytica Posteriora and Metaphysics, with Cleary (1985) arguing that the underlying meaning in his use of the term is subtraction,

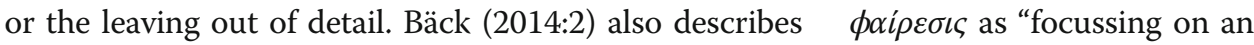
aspect, typically the central one [...] while ignoring the remaining ones." A further historical example comes from Frege's (1884) discussion of abstract objects in mathematics, dubbed the way of negation by Lewis (1986), which involved defining abstract objects as those which lack certain features possessed by their concrete counterparts: spatial and temporal extension, and causal powers. In all of these accounts, there are common themes of omission: either abstract descriptions ignore features, or lack certain properties found in more concrete examples.

From a more modern perspective, Jones (2005) provides a means of distinguishing between two related concepts: abstraction and idealisation. On his account, abstraction involves "mere omission" (ibid: 174), or the omission of truth. Idealisation, on the other hand, is an assertion of a falsehood; it involves claiming that a phenomenon has certain qualities which it in fact does not, or vice-versa. As an example, Jones (ibid: 181-184) considers a model used to predict and explain the trajectory of a ball fired from a cannon. The final resting place of the ball is determined by considering factors such as the angle of trajectory, the force with which the ball is fired, and the effects of gravity. He points to several idealisations and several possible abstractions in models of this kind. Idealisations include: supposing that the ground is flat, that the force of gravity on the ball is equal at all points, and that gravity is the only force affecting the ball after its initial velocity, ignoring factors such as air resistance. Abstractions highlighted include: the colour of the ball, the material constituting it, the ball's internal structure, and its temperature. He argues that in the first list of properties, the modeller is proposing a falsehood, whereas in the latter case she is simply being quiet on certain issues (ibid: 183-184). For example, the abstraction of remaining silent about the colour of the ball is different to an explicit assertion that "the ball has no colour". Compare this to representation of the ground on something like a straight $\mathrm{x}$-axis on a graph. This is an explicit assertion of a fact that we know not to be the case, and this therefore has an impact, however minor, on the predictions made by the model.

This idea of remaining silent without proposing a falsehood is the definition of a "mere omission". This view of abstraction has influenced Godfrey-Smith's (2009) discussion of abstraction in evolutionary biology. He describes abstraction as ignoring detail, whereas idealisation involves an act of imagination; scientists imagine a phenomenon to be different to how we know it to be in reality, which is a clear parallel of Jones' proposal. 
As presented above, all explicit misrepresentations count as idealisations, and every omission of a truth counts as an abstraction. However, these characterisations would cast the net too wide. There would be certain false ascriptions that we would not want to properly count as idealisations, but instead as mistakes. Phlogiston, a postulated constituent of combustible objects believed to exist in the $17^{\text {th }}$ and $18^{\text {th }}$ centuries, acts as an example of an error in a model (Jones, 2005: 186). Additionally, there may be cases where a researcher remains silent on certain issues that are, in fact, crucial to understanding the system under study. In this case, then we may not want to call such omissions abstractions.

The notion of relevancy provides a good way of narrowing down the definitions. Cartwright (1989: 187) and McMullin (1985: 258) both argue that abstraction only occurs in cases where irrelevant facts about the system under study are omitted. We can see links here as well to one of the meanings of abstraction identified by Saitta and Zucker (2013), through the link to the retention of relevant information, and by extension, the omission of irrelevant information. Jones (op. cit.: 190) alters this perspective slightly, by arguing that the term can refer to the omission of relevant factors, but only in cases where this occurs on some idealised basis, that is, factors that seem irrelevant in the model, whereas in the real case they are not. With these subtleties in mind, this work will define one facet of abstraction as the omission of irrelevant information, but not in cases where this amounts to an explicit misrepresentation of the system (which would be called an idealisation).

\section{Abstraction and generalisation}

Another common idea associated with abstraction is that it involves the retention of common features between objects. Again in Aristotle, a potential consequence attributed to this process was the discovery of a universal, which he defined as "that which is common to many individuals" (Coniglione 2004: 60). Lewis (1986) defines his way of abstraction as forming categories by means of comparing a number of experienced objects and only focussing on similarities. This means of comparing and focussing on similarities will, in this work, be taken to be indicative of the process of generalisation.

Generalisation is important to many disciplines, and many discussions of abstraction often involve or imply it. For instance, Ferrari (2003: 1226) writes:

A good share of mathematicians regard abstraction mainly as generalization. The following definition is taken from Wells's (2002: p. 17) online glossary of mathematics:

An abstraction of a concept $C$ is a concept $C^{\prime}$ that includes all instances of $C$ and that is constructed by taking as axioms certain statements that are true for all instances of $C$.

Above, it can be seen that: (a.) abstraction is explicitly linked to the notion of generalisation, and (b.) this process is defined as a set of statements that hold for all members of a particular group of objects. On Ferrari's own account, part of abstraction (but not the only aspect) can be conceptualised as increased inclusion. For instance, the concept of QUADRILATERAL is considered to be more abstract than that of the concept SQUARE, because the former not only contains all instances of the latter but also others such as Rectangle and Rhombus. Therefore, it has as content the aspect that is common to all of these geometrical shapes, while not including the differences between them. In this regard, it generalises over them. 
Mitchelmore and White (2004) name this variant of abstraction empirical abstraction, and discuss its application in the learning of mathematical concepts. They highlight three examples of concepts that children need to become proficient with: addition, angles, and rate of change. In all of these cases, the authors outline a pedagogical strategy whereby students first encounter the relevant mathematical concepts in exemplar settings. After repeated experience of the same concept in a variety of individual settings, children are then able to notice the similarities, and thus grasp the abstract mathematical concept. Mitchelmore and White link this process to the account of abstraction given by Skemp (1986), who describes it as "an activity by which we become aware of similarities [...] among our experiences" (ibid: 21, cited in Mitchelmore and White, op cit: 332).

It should be noted that although generalisation has been discussed so far in terms of the retention of common features, certain accounts of the process have allowed for a slightly weaker reading. Instead of an account of generalisation in terms of necessary and sufficient conditions (e.g. an object $\mathrm{x}$ is a QUADRILATERAL if and only if it is a geometric shape with four sides), Ponsen et al. (2010: 6), in a discussion of computer science, allow for "a weaker definition of generalisation [that] states we have good evidence that all [objects] behave in a similar way." There is a shift here from absolute criteria to a more fluid notion: generalisation is a process that groups certain entities because they are highly likely to exhibit certain features.

Undoubtedly, there are similarities between the processes of generalisation and omission. In choosing to generalise and focus upon the similarities, it is necessary to leave other properties out (since if two objects shared exactly the same set of properties, they would count as the same object). One might be tempted to think, therefore, that generalisation and omission are simply labels that refer to the same underlying process, but simply from differing perspectives. However, this work takes the perspective that although they are closely related, they should properly be kept apart. We argue that: (a.) the two terms are not co-extensive, i.e. that there are cases where only one of the terms can be properly applied, and (b.) the notion of relevancy is part of the meaning of omission, but not of generalisation. Such justification is important, because below in $₫ 4$ these terms will be used as separate criteria in our discussion of theoretical dimensions in SFL.

For the first point, consider the minimum number of individuals (defined here in its broadest sense to include everything from concrete objects to abstract theoretical constructs) that each of the processes operates over. From the above characterisation of generalisation, it is clear that the process must operate over at least two individuals, for means of comparison. Furthermore, the representation that arises out of generalising stands for all of the individuals that were part of the comparison process. The same cannot be said for the related process of mere omission. Instead, omission involves reducing the number of features used to describe a minimum of one individual. This would still hold in cases where another object was used for comparison in order to hypothesise what features are irrelevant, and therefore should be omitted. The consequence is that the representation of the individual that resulted would not hold for the one used for comparison. Note the differences here between the two processes, where, for generalisation, the representation stands for all involved individuals.

Secondly, recall the notion of relevancy, which was argued to be important in the case of feature omission above. Generalisation is only the retention of common 
properties, with no regard to whether those properties that remain are relevant or not. It is of course hoped that the generalised properties will be relevant, but this is not guaranteed by the process of generalisation itself. Strictly speaking, generalisation is 'blind' to the notion of relevancy: it can only arise out of serendipity. Furthermore, properties that may be omitted during an abstraction may indeed be ones that are common to all of the individuals. For instance, in Jones' example, such a model could have been formed by comparing the flights of cannonballs that were all red in colour. Although this property would be common to each individual situation, it could still be the target of the omission process if there was good reason to believe that it is an irrelevant feature of the situation.

Therefore, we believe we are justified in keeping these terms as separate possible aspects of abstraction, and therefore they will be discussed separately when we turn to theoretical dimensions in SFL.

\section{Abstraction and Decontextualisation}

The final aspect to be discussed is the idea of decontextualisation. Some accounts of abstraction highlight a detachment of specific observations and events from their immediate context. For instance, consider Roth (2012: 95, emphasis added):

Science is a successful endeavour because its concepts are applicable to a wider range of phenomena the less they are tied to the specifics of any context, and representations become more inclusive and abstract the less [sic] contextual parameters they include.

Noss et al. (2002: 206) discuss a similar view in terms of what they dub "a traditional view of abstraction." On this view, abstraction is seen to be an "essential property extracted from a situation, but not contained in it," and "deemed 'apart' from, even above, the situation of its genesis" (ibid). This idea of abstractions not being tied to any particular situation is intuitive. After all, when we consider, say, an equation denoting a physical law, or an economic trend, there is not one specific context or situation that is attached to those abstract objects, even the situation(s) that first led to their creation. Instead, they have transcended such specific situations which are thought to hold for all past, present, and (possible) future situations where the circumstances they describe hold.

A concrete theoretical object, then, is one that is directly linked to one particular situation. If we were to create a representation of a particular individual, such as an equation that describes a particular relationship in a particular moment, then this is contextualised. Decontextualised, and thus abstract, theoretical objects are those for which this precise link to a particular situation does not hold.

As with the previous section, it can be difficult to ascertain where two proposed different facets of a concept really should be counted as such. Therefore, it is again necessary to show how decontextualisation is distinct from both omission and generalisation. On face value, there seems to be an immediate distinction to be made between decontextualisation and omission. Omission, defined as omitting features of a system in its description, does not entail a form of decontextualisation; the description, after all, is still representing the original case. Of course, these more abstract descriptions can later be used to cover other situations than the original one. However, such 
extension is not integral to the omission process; it is instead a further kind of abstraction taking place upon an already abstracted object. In the case of generalisation, a similar situation emerges. Given the previously discussed definitions, generalisation simply involves a comparison of two or more objects. Again, this does not entail any decontextualisation. It is possible for someone to provide a description of two objects directly in front of her. That description is then directly linked to that situation; it is general, but contextual.

\section{Summary}

This section has overviewed some of the common aspects that are associated with the term abstraction in academic practice. The characteristics focussed upon can be summed up as follows:

Abstraction as Omission: A move in abstraction can involve the omission of features of a system. Such abstraction should not involve explicit misrepresentation of the phenomenon under study and the omitted features should be irrelevant for the task at hand.

Abstraction as Generalisation: A move in abstraction can involve generalising over more than one concrete counterpart, and retaining what is common between them.

Abstraction as Decontextualisation: A move in abstraction can involve a degree of detachment from situations associated with more concrete counterparts. A more abstract description will be tied to a greater number of cases than a less abstract one.

It should be noted that the above should not be seen as mutually exclusive choices. In most cases, more than one of the above characterisations will be involved, and often there are aspects of all three. As will be shown in the next section, the theoretical architecture of SFL does employ all three of the above aspects, and these different kinds of abstraction can be linked to different theoretical dimensions.

\section{Abstraction in systemic functional linguistics}

With the above three criteria outlined, we now provide a more explicit exposition of the term abstraction in SFL with respect to the theoretical dimensions of delicacy, instantiation and stratification.

\section{Delicacy}

The most important modelling tool within SFL is the system network. It allows the linguist to outline the structure of the various linguistic systems that are deemed important in considering language as a social semiotic. In doing so, the system network signals the prominence that SFL gives to paradigmatic relations between linguistic categories. The inherent ordering along the scale of delicacy allows paradigmatic distinctions to be structured according to a scale of specificity; the network ranges from the more general to the more specific distinctions that can be made between different types of linguistic structures, be they phonological, lexico-grammatical, or (discourse) semantic. This dimension has achieved importance through the notion of the "grammarian's dream" (Halliday 1961; Hasan 1987): the idea that grammar and lexis are not two distinct phenomena, but instead lexis should be seen as the most delicate grammar.

In $\mathbb{\$} 1$, it was noted that delicacy has been linked to the notion of abstraction, especially in Halliday (1961) and Halliday and Matthiessen (1999). In this section, this link will be interpreted as in Fig. 1 below: 


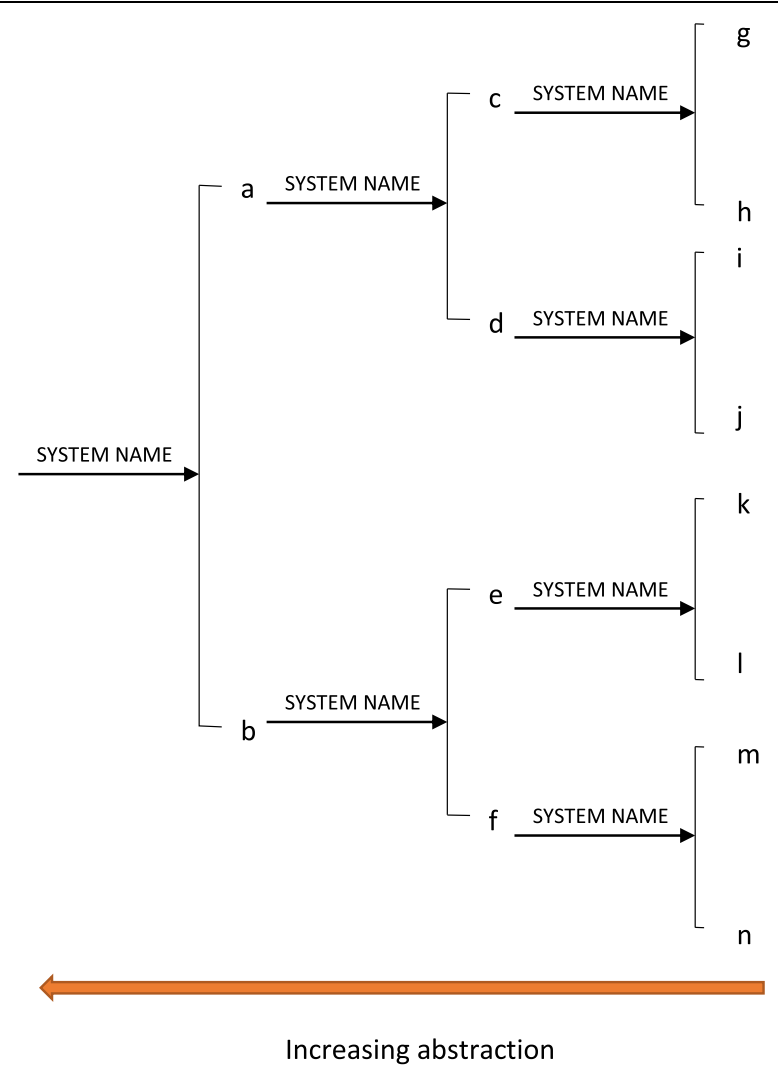

Fig. 1 Abstraction and the scale of delicacy. System features to the left are seen as more abstract than the more specific system features that they dominate

Taking the well-known lexicogrammatical system network of MOOD TYPE as an example, a system feature such as indicative would count as more abstract than those it dominates (declarative and interrogative), and indeed a move from one level of delicacy to another immediately preceding it would be seen as a move in abstraction.

The first aspect to consider is whether a move in abstraction along a system network amounts to the omission of information, without the explicit misrepresentation of the phenomenon under study. Initially, we will discuss the issue of loss of information before turning to the question concerning misrepresentation. In order to discuss these points, this discussion will make use of the system of MODALITY TYPE, one of the four simultaneous systems that make up the overall system of MoDALITY, as stated in Halliday and Matthiessen (2014: 182). At a general level of delicacy, this system distinguishes between two modality types: modalization and modulation. At a further level of delicacy, the system of MODALIZATION is divided into probability and usuality, while distinctions are made between obligation and inclination within the system of MODULATION. For sake of exposition, these options will be applied to the sentences given below:

(1a.) It is likely that John's car keys are in his coat pocket.

(b.) Typically, the lab members are in the pub on Friday evenings.

(c.) I can drive you to the airport tomorrow afternoon.

(d.) I have to meet my boss for a meeting later today. 
Analysing these sentences according to the less delicate (and thus more abstract) option, the following distinction can be made:

(2) MODALizATiOn $=(1 \mathrm{a}$.$) and (1 \mathrm{~b}$.

(3) MODULATION $=(1 \mathrm{c}$.$) and (1 \mathrm{~d}$.)

That is, at this level of delicacy, the sentences that are grouped together are described in the same way. ${ }^{2}$ When we make more delicate choices, and thus less abstract choices, differences between these sentences emerge:

(4a.) MODALIZATION - PROBABILITY $=(1 \mathrm{a}$.

(b.) MODALIZATION - USUALITY $=(1 \mathrm{~b}$. $)$

(c.) MODULATION - OBLIGATION $=(1 \mathrm{c}$. $)$

(d.) MODULATION - INCLINATION $=(1 \mathrm{~d}$. $)$

It is clear that the descriptions of the sentences in (2) and (3) have omitted some information that is included in the representation of the same group of sentences in (4). How else could we get a situation where the same sentences are treated as identical in one case, but then different in another? The less delicate, and thus more abstract, description must be omitting some information that is included in its more delicate, and thus more concrete, counterpart. To take (2), the more abstract description simply attributes that property of specifying a certain degree of commitment to the proposition to these sentences, for this is the definition of what it is to be a member of the category MODALIZATION (Halliday and Matthiessen 2014: 177). ${ }^{3}$ In the more delicate cases (4a). and (b.), (1a.) has the property of being "equivalent to 'either yes or no' [...] with different degrees of likelihood attached" (ibid), while (1b.) has the property of being "equivalent to 'both yes or no', i.e. sometimes yes, sometimes no, with different degrees of oftenness attached." (ibid). For this reason, they are labelled as PROBABILITY and USUALITY respectively. This additional information leading to this distinction is omitted in the description in (2), otherwise we would have a situation where the description is claiming that two sentences with different relevant (lexicogrammatical) features are members of the same (lexicogrammatical) category.

Having shown that information is omitted in a more abstract point of delicacy, it is now necessary to determine whether this omission amounts to explicit misrepresentation. In other words, when describing a text at a certain point in delicacy, does this amount to an assertion that the text lacks the properties associated with the more delicate system features, or alternatively an act of remaining silent with an implicit ascertain that such options are irrelevant for the task at hand? The latter option seems closer, we think, to the spirit of systemic description. Halliday and Matthiessen (1999: 325-326), for instance, discuss delicacy in the following way: pitching an analysis at a certain level of delicacy is a conscious choice on the part of the linguist that should take into account the use that can be made out of that description. There are dangers associated with analysing texts at a certain point in delicacy: an analysis that is too general risks glossing over potentially illuminating distinctions, and one that is too specific risks obscuring meaningful patterns and similarities in the data. These pragmatic considerations motivate the view that omitting detail in more abstract descriptions amounts not to an explicit misrepresentation, but an act of remaining silent on certain 
features of the text, since they are deemed to be irrelevant to the task of the linguist. Therefore, delicacy does include the notion of abstraction as omission.

A discussion of generalisation with regards to delicacy seems to be a relatively easy task; after all, the notions of 'general' and 'specific' features are bound up in the very notion of delicacy. On closer inspection, this initial link is justified, since it is the case that less delicate descriptions contain the information that is common between the more delicate options. This can be seen above, where the less delicate category of MODALIZATION retains the feature that is common to both (1a.) and (1b.): the property of specifying a level of commitment to a proposition. Or take another example from the overall system of MOOD, that of MOOD TYPE. The more abstract feature of indicative specifies that the sentence contains both a Subject and a Finite, and that this property is common to all of the more delicate system features that it dominates, be they interrogative or declarative. Whatever differentiates more delicate options, the properties that define the more abstract category must be inherited by them all (Halliday and Matthiessen 1999: 326). In this way, the dimension of delicacy generalises over texts, and therefore abstraction in this case carries this meaning.

The question of whether a move in decreasing delicacy involves decontextualisation is a subtle one, but we believe that the correct answer is in the negative. The complicating factor is that it at first seems obvious that a less delicate point like indicative will describe a larger amount of cases than a more delicate feature that it dominates, like declarative. It might seem tempting to argue that this fact results in delicacy involving the notion of decontextualisation, given the summary at the end of the last section.

However, this initially attractive view can be refuted by remembering that the overall function of the system network is to describe the potential for meaning making activity in an aspect of a given language. The system network, then, divides up this potential in different ways according to the pragmatic considerations discussed earlier; within the overall potential of linguistic meaning making, there are many ways of dividing up this potential depending on what the linguist is intending to examine. Considering the lexicogrammatical potential of language, the most abstract way this potential can be divided up is in three: into the simultaneous systems of TRANSITIVITY, MOOD, and THEME. Many years of linguistic description and hypothesising has told us that there are many more ways to divide up this potential even further. SFL scholars represent these means of further divisions in system networks. However, regardless of the number of ways this potential is split, it still involves the same linguistic capability. The question, then, is not whether a less delicate system feature like indicative is more removed from a situation than a more delicate option like interrogative, but instead the comparison should be made between indicative and all of the system features it dominates. Put in this way, it is clear that the same underlying potential is being described, and therefore these two levels of abstraction are at the same level of decontextualisation. We feel justified, therefore, in not including this aspect of abstraction in the meaning of delicacy.

\section{Instantiation}

The cline of instantiation links individual texts with the overall system of language. Text is defined as any particular example of language, either spoken or written, which forms a unified whole and carries meaning (Halliday and Hasan 1976: 1-2). The 
linguistic system is conceptualised as a potential for meaning-making linguistic activity. As mentioned in $\$ 1$, links between instantiation and abstraction have been made, especially by Matthiessen (2012: 450). In this section, we will follow Matthiessen's lead, and interpret abstraction as applying to this dimension as in Fig. 2 below:

The major theoretical view expressed by the cline of instantiation is that there is no fundamental difference between linguistic texts and the underlying system of language: potential and instance are simply two different viewpoints taken upon the same phenomenon (Halliday 1992b.: 27; Halliday and Matthiessen 1999: 328). The usual analogy made is that of weather and climate. Weather describes meteorological patterns on a short-term, daily basis; climate describes the same kind of patterns, but instead over a longer time-scale. However, they are fundamentally describing the same phenomenon. The view that system and text are not two distinct phenomena differs from the distinction between langue/parole of Saussure, and the dichotomy between competence and performance in generative linguistics.

This relationship between system and instance is envisioned as a cline, thus incorporating a number of intermediary points between the two. These mid-points can be considered in two ways: either they are text types (viewed from the instance end) or as subpotentials/domain models within the overall meaning making potential of the linguistic system (viewed from the system end). ${ }^{4}$ At the stratum of the context, the terms situation types and institution are used to indicate the same notions. The 'dual-perspective' nature of the cline of instantiation is fundamental to understanding the theoretical importance of this dimension.

An example will help elucidate these ideas further. Halliday and Matthiessen (1999: $\$ 8.3$ ) provide a description of the domain model of cooking recipes at the stratum of semantics. More specifically, the authors describe this particular domain model as a particular variant of the overall ideational semantic model presented in their work. Within this domain model, a number of characteristics are identified:

1. Absence of 'metathings' such as facts, ideas or locations

2. A higher proportion of figures of doing; lower proportion of figures of sensing and being.

3. No figures of saying (in data set considered, although not ruled out as a possibility).

4. Sole appearance of sequences of expansion, with no (or very occasional) examples of projecting sequences.

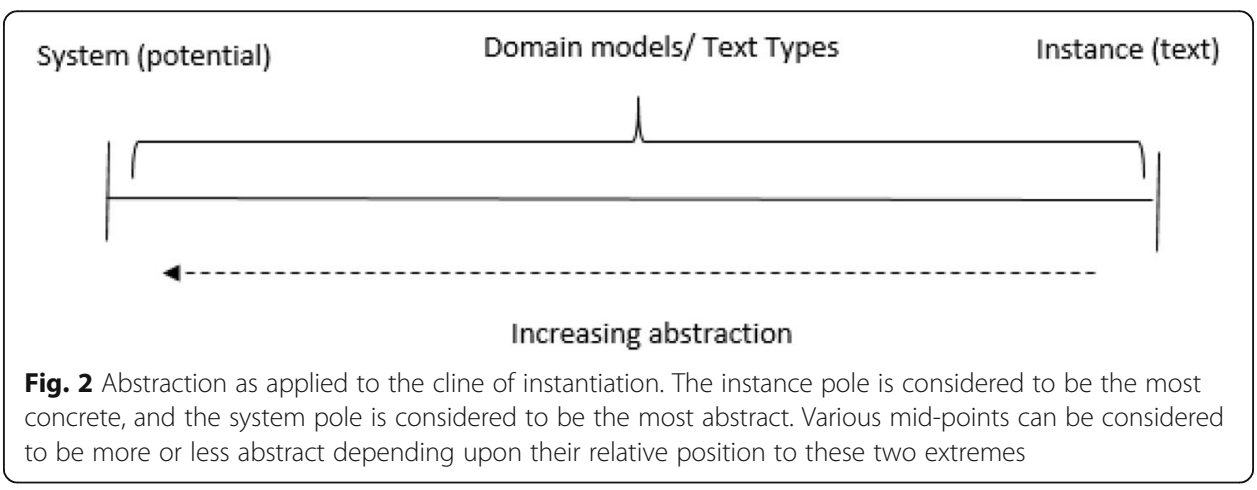


In this case, the two major types of variation mentioned above are evident. In the domain model, some system features included in the overall model of ideational semantics are not found, and others appear with a greater or lesser frequency.

The notion of abstraction with regards to the cline of instantiation involves discussion of how we move from the instance end of the cline towards the system end. In other words, it is concerned with how we move from concrete instances of text to the more abstract notions of domain models and the overall system of language.

Departing from the order of the discussion in the last section, it is necessary to begin our discussion of the term abstraction here as it relates to the idea of generalisation. Although the cline of instantiation has been linked to abstraction, the term generalisation has also been used to describe it (Martin 2009: 558; Matthiessen et al. 2010: 121-122). As seen, generalisation forms one of the main meanings of abstraction, so making the distinction between abstraction and generalisation does not necessarily mean that two distinct processes are being referred to.

Halliday and Matthiessen (1999: 323) describe a domain model as a "generalisation across particular instances of text [when] seen from the instance end". Therefore, if the meaning of the term in SFL coincides with the way it is used in other disciplines, a domain model should contain only those properties which are shared by all instances of text that are claimed to be an example of that domain. Focussing on the domain model of cooking recipes described above, it seems as though the use of the term generalization as intended by SFL practitioners differs from this definition. In order to show this, let's turn to the second and third characteristics of this domain model as stated above. We take this characteristic to mean, and this interpretation seems reasonable, that considering all instances of this particular domain: all instances will contain figures of doing, some instances will contain figures of sensing and/or being, but some instances will contain neither, and only a few will contain figures of saying. Therefore, the only property which will be common to all these texts would be the presence of figures of doing, and if a domain model is considered to be a generalization over texts in the sense described above, then only this system feature should be present in the more abstract description.

This is not what SFL practitioners intend when defining a mid-point on the cline of instantiation. The term in this case is to be defined as "an accumulation of frequencies in text" (Wu 2000: 246). In other words, generalisation as it is used here is approached in probabilistic terms, and is considered to be a form of statistical induction. This is still a form of generalisation, although it differs from the kind of generalisation which is usually associated with abstraction. It instead resonates better with Ponsen et al.'s (2010) weaker approach, discussed above.

The ways in which the cline of instantiation correlates with the notion of decontextualisation mainly stem from consideration of points upon this dimension as potentials, as well as generalisations across texts. The notion of potentials is fundamental in systemic theorising, because it allows the linguistic model to apply not just to previously encountered linguistic utterances, but also to additionally try to explain how speakers produce and interpret novel instances of language. Without this conceptualisation, there is a danger of language being considered purely a generalisation over previously encountered texts, a view deemed inadequate for linguistic theory ever since Chomsky's (1959) criticism of behaviourist psychology as applied to language. To see how decontextualisation links to abstraction along the cline of instantiation, let's take three points along this cline: 
firstly, a particular text found at the instance pole, secondly, the domain model of cooking recipes already introduced, and lastly, the overall description of language as system. If the notion of decontextualization applies here, then the concrete example (instance/text) should be more closely linked to a particular context than either the domain model or the overall systemic potential, and the overall system model should be more decontextualized than the domain model. A text is produced, and often considered, within a particular context of situation. Indeed, its character is shaped in part from the situation the text is produced in and the relevant configurations of field, tenor and mode variables. As such, a text is fully contextualized. This is exemplified by the fact that texts are considered to be linguistic tokens (Halliday and Matthiessen 1999: 15). The system, on the other hand, represents the entire meaning making potential of languages. As such, it is not tied to any particular setting, but intended, theoretically, to cover all encountered situations and provides the means to successfully function linguistically in any new situation and relates them to the system as already conceived. Although the notion of system of a particular language itself cannot ever be considered to be fully decontextualized, since it has been shaped by the culture of the language users, it involves a higher degree of decontextualization than a particular instance of language. Intermediate points, lying between the two, are more removed from specific contexts than a text, but less so than the overall system. In the example already mentioned, the domain model of cooking recipes can be viewed as a variant of the overall ideational semantic potential of language. It is a characterization of the potential of creating meaning within a certain specified setting; in other words, its characterization is field motivated (as the ideational metafunction correlates with the contextual metafunction of field, see Hasan 1995; Thompson 1999). It is the potential of creating meaningful text in a restricted set of circumstances; it is not tied to any specified on particular context, but instead is a characterization of the relevant aspects of the ideational potential which are activated in a set of circumstances of a particular type. Therefore, it is more contextualized than the overall system, which must cover all potential instances of language use, but less contextualized than a text, which is intimately tied to the particular setting in which it was created. As a result, instantiational abstraction involves a degree of decontextualization as part of its meaning.

Unlike the dimension of delicacy, abstraction along the cline of instantiation does not, we claim, include the omission of any factors found at the concrete end of the cline. This difference stems from the types of generalisation that operate in each case. As was discussed in the last section, omission was evident along the scale of delicacy because more abstract features in system networks omit features which are present in their more concrete counterparts. It was linked to generalization, because the same process can be described as the focus on the similarities between two or more concrete features. As just discussed, generalization along the cline of instantiation operates more in terms of a statistical induction; on the basis of past experience, the linguist builds up a statistical prediction of what features are likely to be exhibited by a particular text. This is most clearly seen in domain models. The question now remains: do the more abstract points on the cline omit any (irrelevant) information that is found in their concrete counterparts, i.e. the set of texts that they are statistically generalizing over? The answer seems to be no. For example, we can imagine a text with a figure of saying found in the creation of the cooking recipe domain model (which was not attested to in the sample given, but was thought to be a possibility). In this case, the variant of the 
ideational potential which defines that domain model must include the possibility of figures of saying, but it would note its relative rarity by attributing to it an extremely low probability of occurring. Similar processes would apply to other rare and atypical text features at all the strata which make up the SFL model. As a result, abstraction as applied to the cline of instantiation does not carry the meaning of omission.

\section{Stratification}

As mentioned at the beginning of this paper, stratification is most frequently discussed explicitly in terms of abstraction, with the higher strata considered to be more abstract than those positioned below them. Figure 3 shows the means by which the stratificational dimension is usually diagrammed (following Martin and Matthiessen, 1991) and how the notion of abstraction fits into the framework:

Stratification allows researchers to "talk about language under different headings" (Halliday and Matthiessen 2014: 24). Language can be described as a system of 'sounding' (phonology), a system of 'wording' (lexicogrammar), or a system of 'meaning' (semantics). Indeed, language should be viewed as being all of these things, because language as modelled in the instantiation cline operates at all of these strata simultaneously (Halliday 2002: 19; Martin 2009: 559). As we move from phonology, to lexicogrammar, and on to semantics and context, we discuss language in increasingly abstract terms.

If the characterisation of abstraction involving omission is accurate in this case, then the following two features should be evident: firstly, that the more concrete strata should contain more information than the more abstract ones; and secondly, the information at the higher strata should be derived from the lower ones, on the basis that they are formed by the omission of factors which are deemed irrelevant as a move is made from the concrete to the abstract. The concept of abstraction in the stratificational model fulfils neither of these criteria.

The notion of system at each of the perspectives in the model indeed includes the omission of detail, but what is omitted in each case is not derived from the strata immediately below. For example, at the strata of lexicogrammar, the linguist is interested in viewing language as a system of wording and as such, the categories which are relevant at this

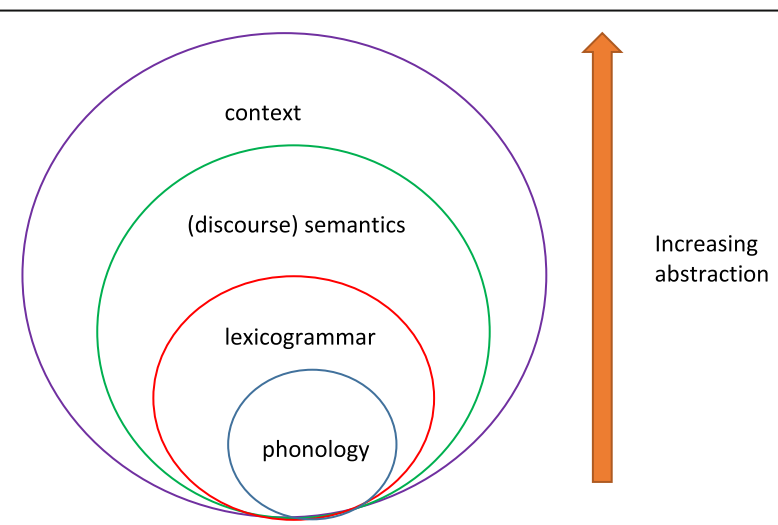

Fig. 3 Abstraction with reference to stratification. Higher strata are deemed to be more abstraction than those below them 
stratum are those relating to the grammatical properties of language. For instance, the grammatical system of MOOD TYPE contains system features such as indicative, imperative, interrogative and declarative, and they are realized (inter-stratally) via relations between grammatical units (Subject, Finite, etc.). Consider what would be the case if the lexicogrammatical system was abstract in the sense that it omitted details that were present in the more concrete stratum of phonology. In this case, the features which were relevant for grammatical analysis would have to be present in the phonology, in order to also occur at the higher stratum. Phonology, therefore, would have to be a description of language as sounding and wording. This clearly goes against what phonology is typically taken to be. It would also run contrary to the arbitrary relationship between the production plane of phonology and the higher, more abstract, content plane (c.f. Martin 1992: 20; Halliday and Matthiessen 1999: 5; Taverniers 2011), since if the information relevant at the lexicogrammatical level was present in its own production plane, then we lose the justification for calling the relationship between these two strata arbitrary. Similar observations would hold between the other strata. In order for the process of abstraction to be construed in terms of mere omission in this case, all relevant information for the study of language must be present at the most concrete level, and each subsequent move in abstraction would reduce the amount of information in an incremental matter, with the only retained information being kept in each case. This is not the process we see for stratification, possibly motivating Halliday to consider the description of language at each stratum to be a description according to different types (Halliday 1981 [2005]).

The idea of links between strata being considered as a type of generalization has previously been offered by Martin and Matthiessen (1991: $\$ 2.5)$. Interesting for our purposes here is that they consider the more concrete strata to be more general than the more abstract ones. This is clearly contrary to the idea of generalization as we have been considering it so far, which has taken generalization to be associated with more abstract constructs, not with more concrete ones. ${ }^{5}$ They argue for this 'downward' generalization on the observation that multiple (sub-) systems at a higher stratum can be realized by a lower number of (sub-) systems at the level immediately below it. For instance, they give the example of the KEY system network at the stratum of lexicogrammar, and the system of TONE at the phonological stratum. The two KEY systems which are relevant to the system network of MOOD TYPE (one for the declarative option, and another for the interrogative option within the system network of MOOD TYPE) are realized by the one system of TONE. In other words, in their view, system networks at the more concrete strata can convey a greater amount of information than the system networks at the more abstract strata.

Assuming that Martin and Matthiessen are correct in their account of the relationship between strata, we will examine their concept of generalization in this case. It is clear that it is not based on statistical inference of relative frequency of tokens, since the system networks are themselves relations between linguistic types. Neither is the TONE system a generalization of the system of KEY in the sense seen in the dimension of delicacy. The reason for this is subtle, but important. In order to ground this discussion, let's refer to the following two short sentences (examples based on or taken from Halliday and Greaves 2008):

(5) Is that you?

(6) I like it! (challenging: 'what makes you think I don't?)

Example (5) above is a simple unmarked yes/no interrogative, and (6) is an example of a type of marked declarative associated with contradicting information just presented 
to the speaker. Both of these are realized at the phonological system of TONE by a rising intonation (tone 2). The observation by Martin and Matthiessen is that options in two distinct system networks (DeClarative and inTERrogative, themselves more delicate systems of the superordinate system of MOOD TYPE) are realized by only one system at the stratum below. However, this is not a generalization as retention of similarities of the lexicogrammatical properties of the two sentences above. They are not generalizations of the above two sentences as instances of wording, they are generalizations of these sentences as instances of sounding, information not relevant at the stratum of lexicogrammar given the epistemological approach of stratification that forms the basis of this dimension of functional linguistic study.

Neither does the notion of abstraction in the stratificational model imply a move in terms of level of contextualisation. The reason for this is relatively straight forward. If phonology was more concrete than lexicogrammar in this sense, then the notion of phonology itself would be more tied to particular contexts than lexicogrammar or semantics, which does not seem to be the case. For illustration, let's consider the strata of phonology and lexicogrammar and fix them at the system end of the cline of instantiation, so as to negate any influences this independent dimension may have on our thinking. Considering the two strata as the system of language viewed at different levels of abstraction, it cannot be the case that one or the other of these are strata are more or less contextualized than the others. Both the description of the meaning potential of language at the phonological stratum and the lexicogrammatical stratum are meant to account for all the possible meanings that people can make using linguistic resources. If this was not the case, then (considering spoken language) one would expect to find actual instances of language use which exhibit grammatical properties but not phonological ones. The same would hold between grammatical and semantic properties, however defined. We take such a view to be implausible. Therefore, abstraction as it applies to stratal movement does not include a higher or lower level of contextualization.

\section{A typology of abstraction types in SFL}

To summarise the discussion in this section, Table 1 provides the differing meanings of abstraction that have been argued to be evident in SFL with respect to the three characteristics of this notion identified earlier:

From this table, we can see that all three of the commonly discussed features of abstraction find their way into the overall theory of SFL. Both delicacy and instantiation allow us to generalise over texts, albeit in slightly different ways. Delicacy additionally allows us to omit detail from our analyses, and thus permits patterns to emerge that may otherwise remain hidden. Instantiation, on the other hand, allows us to decontextualise our theory of language, by allowing us to move from the text to the system.

Table 1 The differing meanings of abstraction in Systemic Functional Linguistics

\begin{tabular}{llll}
\hline & Mere Omission & Generalisation & Decontextualisation \\
\hline Delicacy & $\checkmark$ & $\checkmark$ & $\boldsymbol{x}$ \\
Instantiation & $\boldsymbol{x}$ & $\checkmark$ & $\checkmark$ \\
Stratification & $\boldsymbol{x}$ & $\boldsymbol{x}$ & $\boldsymbol{x}$ \\
\hline
\end{tabular}


Stratification, it seems, does not follow the same pattern, as it does not exhibit any of the common features of abstraction identified in this paper. It is worth returning briefly to this dimension within the theoretical architecture, in order to discuss this potentially surprising finding.

\section{Stratification: The problem case}

As has been noted, out of the three theoretical dimensions discussed here, stratification is the one most frequently associated with the term abstraction. However, if the arguments presented here hold, this is the one dimension that does not exhibit any of the common characteristics of abstraction. To reiterate, this does not mean that the term is being used incorrectly, but instead it may have a more idiosyncratic meaning. However, we believe that one consequence of this study is that this different use of the term is in need of further elucidation. This will be of real importance, as it will be at the heart of the explicit ordering between strata that is provided by the dimension of stratification.

A full discussion of stratification must wait for a later time, but we would like to conclude this study by discussing briefly one idea that has been associated with this dimension: supervenience (Martin 2014). In a discussion outlining his intellectual history within SFL, Martin (ibid: 11) states that two views can be taken concerning the link between language and social context: the supervenient and the circumvenient. He argues for the former, based upon the common perspective in the theory of context and language in which they form a connotative semiotic system. Given that these two notions enter a stratificational relationship, with context being a stratum of the semiotic system above the linguistic ones, then it is possible that the idea of supervenience may hold the key to understanding the basis of the stratificational dimension (ibid: 14).

Such a view, if found to be true, would be a benefit to the theory, as supervenience as a means of linking higher and lower structures has received wide-spread discussion since its introduction in philosophical ethics (Hare 1952). Since then, the application of the notion in the philosophy of mind has led to formal accounts of the relationship being proposed (c.f. McLaughlin and Bennett 2014 for a recent review). However, an examination of the properties of supervenience will show that it does not map well onto accounts of stratification.

Supervenience comes in a number of different varieties (Kim, 1984). However, as nothing we will say hinges upon the differences entrenched in these variations, a reasonably broad conceptualisation of supervenience will suffice (the definition below is based upon Davidson 1970: 214):

(7) A set of properties A supervenes upon another set of properties B if and only if two things that are identical to each other in terms of B properties are also identical to each other in terms of A properties.

Another way of considering supervenience is: given a set of properties $\mathrm{A}$, and a different set of properties B, two objects that have different A properties must also have different B properties. Within the philosophy of mind, this is usually used to describe the relation between mental and physical properties: if two objects (say individuals) share the same physical property (i.e. brain states), they must also share the same mental property (i.e. psychological state). The reverse need not hold, allowing for more variety in the lower-level realisation of mental properties. 
Adapting this characterisation into functional linguistics, if the inter-stratal relation can be described in terms of supervenience, then we should see similar dependencies between higher and lower level properties of a text. If we take, for instance, the lexicogrammatical-semantics interface (since this is arguably the most studied case, c.f. Taviniers 2011), then we can consider set A to be the set of higher level semantic properties and set $\mathrm{B}$ to be the set of lower level lexicogrammatical properties. If these two sets of properties are linked by a supervenience relation, then, given (7), the prediction would be that any two texts which share the same set of lexico-grammatical properties, must share the same semantic property. A cursory glance at the nature of inter-stratal relations gives us reason to doubt that this is the case. Such doubt comes from the idea of congruent and metaphorical realisations. When considered, for instance, with reference to semantics and lexicogrammar, a metaphorical realisation refers to a realisation of a semantic feature by atypical means (c.f. Halliday 1985 for the original proposal, and Ravelli 1985 [1999] Taverniers 2003; Devrim 2015 for discussions). In broad strokes, if one accepts that there exist typical and atypical realisations of semantic properties in the lexicogrammar, then it must be the case that it is possible for two texts to be different in their lower-level (lexico-grammatical) properties, but identical in their semantics. This is the situation that supervenience explicitly rules out.

Of course, it could be possible that Martin simply takes the supervenient view to mean that context should be considered as another stratum in line with the language internal ones, without intending supervenience to make reference to the proposals put forward in the philosophical literature. In this case, however, his proposal leaves the ultimate question under discussion in this section unanswered, until a more specific proposal is provided. Additionally, the brief discussion above cannot rule out that some features that are linked by stratification cannot be described in terms of a supervenience relation. For instance, it is possible that context and language are, but the interlanguage strata are not, which one might read Martin as suggesting (although our reading is that he intends this relationship to hold between all strata). In that case we at best have only a partial description of the relation, and at worst have reason to doubt that stratification operates over a unified scale of abstraction. This would have widespread theoretical consequences for the place of context in the model.

Supervenience, then, does not seem to be able to survive as a candidate for the underlying principle for the ordering of linguistic and contextual strata.

\section{Conclusions and future research}

Theoretical terms are important in any discipline, and their usefulness is undoubtedly ubiquitous in academic practice. However, their continuing use is dependent upon a close understanding of their meanings. In this paper, we have attempted to undertake such a task by examining the nature of delicacy, instantiation and stratification within Systemic Functional Linguistics by interrogating the notion of abstraction in each case. Our main conclusion is that although the term abstraction has been evoked in relation to all of them, what is meant by this term differs in each case. With respect to delicacy and instantiation, these meanings map on well to common properties identified by authors who have discussed the issue in other academic disciplines. In the case of stratification, however, there seems to be something else at work. The precise nature of stratification, and the question of what factors determine what it means for a given 
stratum to be at a 'higher' or 'lower' level to another is one we will have to leave for later work. An acceptable answer, however, is of fundamental importance to the field. However, we hope that in this work we have shown the use that can come out of interrogating the theory itself, and that those using the SFL framework in their work will find use in the proposals made concerning the practices that are bound up in these theoretical terms.

\section{Endnotes}

${ }^{1}$ The principle of certus paribus is usually translated as all other things being equal.

${ }^{2}$ At least with respect to this system network. There will undoubtedly be differences in other parts of the grammar. This fact does not affect the argument put forward here. All that needs to be accepted is that the sentences grouped together in (2) and (3) are described identically in this respect.

${ }^{3}$ How best to describe the feature this level of delicacy attributes to these sentences need not concern us too much, as long as the same feature is being applied to both sentences.

${ }^{4}$ In some work (e.g. Matthiessen 2007a: 535) these intermediate points have been called registers. However, register also carries a different meaning in SFL, functioning at the contextual strata, especially in the work of James Martin and David Rose. The term sub-potential will be used here to avoid confusion.

${ }^{5}$ Martin and Matthiessen also argue that generalization applies to the rank scale, using similar arguments to the ones discussed here. This piece has chosen not to focus upon this dimension, as rank is not generally considered to involve a form of abstraction.

Abbreviations

SFL: Systemic Functional Linguistics

\section{Acknowledgements}

This work was presented at the 42nd International Systemic Functional Congress at RWTH University Aachen in 2015. The authors would like to thank all of those in attendance, especially Tom Bartlett, Lise Fontaine, and Mick O'Donnell for their helpful comments on the arguments presented. Thanks also to Nicola Pitchford, Department of Psychology, University of Nottingham, for comments on an earlier draft of this work.

\section{Funding}

This research was conducted under the JW's institutional PhD funding from the Department of Engineering, University of Nottingham, UK, and the Faculty of Arts and Social Sciences, University of Nottingham, Malaysia Campus.

Availability of data and materials

All data presented here is taken from already published sources. The sources of these are provided in the text when mentioned.

\section{Authors' contributions}

The concept for this work was developed by JW. Research was undertaken by all of the authors. Manuscript was prepared by JW. All authors were involved in proof-reading manuscript ready for publication. All authors read and approved the final manuscript.

\section{Authors' information}

This work was undertaken while the first author was a PhD student at the University of Nottingham, and the University of Nottingham, Malaysia Campus. His current contact address is: School of English, Media, and Creative Cultures, Nottingham Trent University, Clifton Lane, Nottingham, NG11 9NS.

Ethics approval and consent to participate

Not applicable

Consent for publication

Not applicable 


\section{Publisher's Note}

Springer Nature remains neutral with regard to jurisdictional claims in published maps and institutional affiliations.

\section{Author details}

${ }^{1}$ School of English, University of Nottingham Malaysia Campus, Jalan Broga, 43500 Semenyih Selangor Dahrul Ehsan, Malaysia. ${ }^{2}$ School of Electrical and Electronic Engineering, University of Nottingham, University Park, Nottingham NG7 2RD, United Kingdom. ${ }^{3}$ School of English, University of Nottingham Ningbo, 199 Taikang E Road, Yinzhou Qu, Ningbo Shi, Zhejiang Sheng 315100, China.

Received: 10 May 2017 Accepted: 7 September 2017

Published online: 19 September 2017

\section{References}

Bäck, Allen. 2014. Aristotle's Theory of abstraction. Dordecht: Springer.

Barsalou, Lawrence W. 2003. Abstraction in perceptual symbol systems. Philosophical Transactions of the Royal Society of London, B (Biological Sciences) 358: 1177-1187.

Barsalou, Lawrence W. 2005. Abstraction as dynamic interpretation in perceptual symbol systems. In Building abstract categories. Carnegie symposium series, ed. L. Gershkopf-Stowe and D. Rakison, 389-431. Majwah, NJ: Erlbaum.

Cartwright, Nancy. 1983. How the Laws of physics lie. Oxford: Oxford University Press.

Cartwright, Nancy. 1989. Nature's Capacities and their measurement. Oxford: Oxford University Press.

Chomsky, Noam. 1959. A review of B.F. Skinner's Verbal Behaviour Language 35 (1): 26-58.

Chomsky, Noam. 1995. The minimalist program. Cambridge, MA: MIT Press.

Cleary, John J. 1985. On the terminology of 'abstraction' in Aristotle. Phronesis 30 (1): 13-45.

Coniglione, Francesco. 2004. Between abstraction and idealization: Scientific practice and philosophical awareness. Poznań Studies in the Philosophy of the Sciences and the Humanities 82: 59-110.

Devrim, Devo Y. 2015. Grammatical metaphor: What do we mean? What exactly are we studying? Functional Linguistics 2 (3). https://doi.org/10.1186/s40554-015-0016-7.

Ferrari, Pier Luigi. 2003. Abstraction in mathematics. Philosophical Transactions of the Royal Society 358: 1225-1316.

Frege, Gottlob. 1884. Die Grundlage der Arithmetik. Translated by J.L. Austin as the foundations of arithmetic. Oxford, Blackwell, 1959.

Godfrey-Smith, Peter. 2009. Abstractions, idealizations and evolutionary biology. In Mapping the future of biology: Evolving concepts and theories, ed. A. Baraberousse, M. Morange, and T. Pradeu, 46-56. Dordecht: Springer Publishing

Halliday, M.A.K. 1961. Categories of the theory of grammar. Word 17 (3): 241-292.

Halliday, M.A.K. 1981 [2005]. Text semantics and clause grammar: How is a text like a clause? Reprinted in: The collected works of M.A.K. Halliday, Vol 1: On grammar, ed. Jonathan J. Webster, 219-261. London, continuum.

Halliday, M.A.K. 1985. Systemic background. In Systemic perspectives on discourse, volume 1. Selected theoretical papers from the $9^{\text {th }}$ international systemic workshop, ed. James D. Benson and William S. Greves, 1-15. Norwood, NJ: Ablex.

Halliday, M.A.K 1992a. [2005]. Systemic Grammar and the Concept of a "Science of Language". Waiguoyu (Journal of Foreign Languages) 2: 1-9. Reprinted in: The Collected Works of M.A.K. Halliday, Vol 3: On Language and Linguistics, ed. Jonathan J. Webster, 2005, 199-212. London: Continuum.

Halliday, M.A.K. 1992b. How do you mean? In Systemic linguistics: Recent theory and practice, ed. Martin Davies and Louise Ravelli, 20-35. London: Pinter.

Halliday, M.A.K. 2002. Computing meanings: Some reflections on past experience and present prospects. In Discourse and language functions, ed. Guowen Huang and Zongyan Wang, 3-25. Shanghai: Foreign Language Teaching and Research Press.

Halliday, M.A.K. 2009. Methods-Techniques-Problems. In Continuum companion to systemic functional linguistics, ed. M.A K. Halliday and Jonathan J. Webster, 59-86. London/New York, NY: Continuum

Halliday, M.A.K., and William Greaves. 2008. Intonation in the grammar of English. London: Equinox.

Halliday, M.A.K., and Ruqaiya Hasan. 1976. Cohesion in English. London: Longman.

Halliday, M.A.K., and Christian M.I.M. Matthiessen. 1999. Construing experience through meaning: A language-based approach to cognition. London/New York: Continuum.

Halliday, M.A.K., and Christian M.I.M. Matthiessen. 2014. Halliday's Introduction to functional grammar. 4th ed. Abingdon: Routledge.

Hare, R.M. 1952. The language of morals. Oxford: Oxford University Press.

Hasan, Ruqaiya. 1987. The grammarian's dream: Lexis as most delicate grammar. In New developments in systemic linguistics. Vol 1, theory and description, ed. M.A.K. Halliday and Robin Fawcett, 184-211. London/New York: Pinter.

Hasan, Ruqaiya. 1995. The conception of context in text. In Discourse in society: Systemic functional perspectives, ed. P. Fries and M. Gregory, 183-283. Norwood, NJ: Ablex.

Hasan, Ruqaiya. 2013. Choice, system, realisation: Describing language as meaning potential. In Systemic functional linguistics: Exploring choice, ed. Lise Fontaine, Tom Bartlett, and Gerard O'Grady, 269-299. Cambridge: Cambridge University Press.

Jones, Martin R. 2005. Idealization and abstraction: A framework. In Idealization XII: Correcting the model: Idealization and abstraction in the sciences (Poznań studies in the philosophy of the sciences and humanities, 86), ed. Martin R. Jones and Nancy Cartwright, 173-217. Amsterdam/New York: Rodopi.

Kim, Jaegwon. 1984. Concepts of Supervenience. Philosophy and Phenomenological Research 45: 153-176.

Lewis, David. 1986. On the plurality of worlds. Oxford: Basil Blackwell.

Martin, James R. 1992. English text: System and structure. Amsterdam/Philadelphia, PA: John Benjamins.

Martin, James R. 2009. Realization, instantiation and individualization: Some thoughts on identity in youth justice conferencing. DELTA 25: 549-583.

Martin, James R. 2014. Evolving systemic functional linguistics: Beyond the clause. Functional Linquistics 1 (3). https://doi. org/10.1186/2196-419X-1-3. 
Martin, James R. and Christian M.I.M. Matthiessen. 1991. Systemic typology and topology. In: Literacy in Social Practices, ed. Francis Christie, 345-383. Darwin: Centre for Studies in Language and Education, Northern Territory University.

Martinez, Sergio F., and Xiang Huang. 2011. Epistemic groundings of abstraction and their cognitive domain. Philosophy of Science 78 (3): 490-511.

Matthiessen, Christian M.I.M. 2007a. The "architecture" of language according to systemic functional theory: developments since the 1970s. In: Continuing Discourse of Language: a functional perspective, volume 2, eds. Ruqaiya Hasan, Christian Matthiessen and Jonathan J. Webster, 505-561. London: Equinox.

Matthiessen, Christian M.I.M. 2007b. Lexicogrammar in systemic functional linguistics: Descriptive and theoretical developments since the 1970s. In: Ruqaiya Hasan, Christian Matthiessen and Jonathan J. Webster (eds.), Continuing discourse of language: A functional perspective, volume 2. London: Equinox, pp. 765-858.

Matthiessen, Christian M.I.M. 2012. Systemic Functional Linguistics as applicable linguistics: social accountability and critical approaches. DELTA [online] 28: pp. 435-471. Available from: http://www.scielo.br/scielo.php?script=sci_ arttext\&pid=S0102-44502012000300002. ISSN 0102-4450. Accessed 24 Sep 2014.

Matthiessen, Christian M.I.M., Kazuhiro Teruya, and Marvin Lam. 2010. Key terms in systemic functional linguistics. London/New York: Continuum.

McLaughlin, Brian and Karen Bennett. 2014. Supervenience. In: Edward N. Zalta (ed.), The Stanford Encyclopaedia of Philosophy (spring 2014 edition). Available from: http://plato.stanford.Edu/archives/spr2014/entries/supervenience/ Accessed 24 Feb 2016.

McMullin, Ernan. 1985. Galilean idealization. Studies in History and Philosophy of Science Part A 16 (3): 247-273.

Mitchelmore, Michael, and Paul White. 2004. Abstraction in mathematics and mathematics learning. Proceedings of the. In 28th Conference of the International Group for the Psychology of mathematics education 3, 329-336.

Noss, Richard, Celia Hoyles, and Stefano Pozzi. 2002. Abstraction in Experitise: A study of nurses conceptions of concentration. Journal for Research in Mathematics Education 33 (3): 204-229.

Ponsen, Marc, Matthew E. Taylor, and Karl Tuyls. 2010. Abstraction and generalization in reinforcement learning: A summary and framework. ALA 2009: International Workshop on Adaptive and Learning Aspects: 1-32. doi: https://doi. org/10.1007/978-3-642-11814-2_1.

Ravelli, Louise Jane. 1985. Metaphor, mode and complexity: An exploration of co-varying patterns. Re-published 1999. Nottingham: Nottingham Trent University.

Roth, Wolff-Michael. 2012. Undoing Decontextualization or how scientists come to understand their own data/graphs. Science Education 97 (1): 80-112.

Saitta, Lorenze, and Jean-Daniel Zucker. 2013. Abstraction in artificial intelligence and complex systems. New York: Springer.

Skemp, Richard. 1986. The psychology of learning mathematics. 2nd ed. Harmondsworth: Penguin.

Taverniers, Miriam. 2011. The syntax-semantics interface in systemic functional grammar: Halliday's Interpretation of the Hjemslevian model of stratification. Journal of Pragmatics 43: 1110-1126.

Thompson, Geoff. 1999. Acting the part: Lexico-grammatical choices and contextual factors. In In text and context in functional linguistics, ed. Mohsen Ghadessy, 101-124. Amsterdam: John Benjamins.

Wu, Canzhong. 2000. Modelling Linguistic Resources: A systemic functional approach. Ms: PhD Thesis, Macquarie University.

\section{Submit your manuscript to a SpringerOpen ${ }^{\circ}$ journal and benefit from:}

- Convenient online submission

- Rigorous peer review

- Open access: articles freely available online

- High visibility within the field

- Retaining the copyright to your article

Submit your next manuscript at $\gg$ springeropen.com 